\title{
Ansiedade em profissionais de saúde durante a pandemia do novo coronavírus: Uma revisão integrativa
}

\author{
Anxiety in healthcare professionals during the new coronavirus pandemic: An integrative review \\ Ansiedad en los profesionales de la salud durante la nueva pandemia de coronavirus: Una revisión \\ integradora
}

Recebido: 08/07/2021 | Revisado: 14/07/2021 | Aceito: 16/07/2021 | Publicado: 25/07/2021

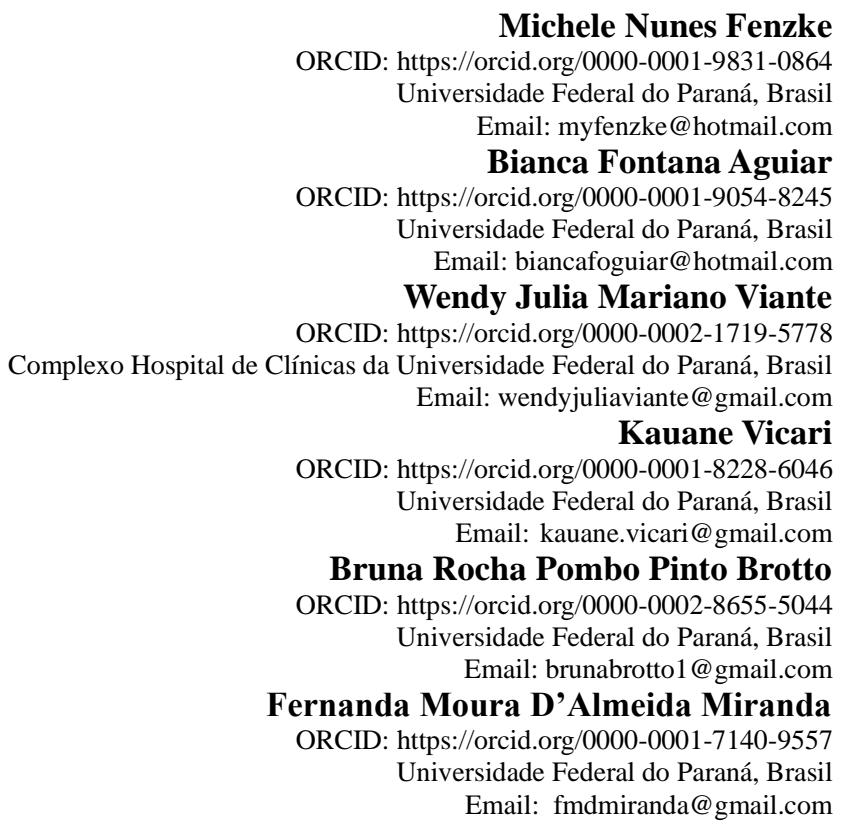

\section{Resumo}

Objetivo: Caracterizar na literatura científica estudos sobre a ansiedade em profissionais de saúde atuantes no combate a pandemia do novo coronavírus e identificar as principais alterações fisiológicas e psicológicas entre os profissionais da saúde atuantes na pandemia da Covid-19. Método: Trata-se de revisão integrativa, sendo a busca realizada em janeiro de 2021, nas bases de dados: LILACS, MEDLINE e BDENF. Usaram-se os descritores Anxiety, Coronavirus Infections e Health Personnel e os critérios de inclusão foram artigos completos, nos idiomas português, inglês e espanhol, publicados entre 2019 a 2021. Optou-se por excluir revisões de literatura e artigos de opinião. Resultados: Foram analisados 50 artigos. Os participantes foram enfermeiros (50\%); médicos (26\%) e outros trabalhadores de saúde (24\%). A maioria das participantes eram do sexo feminino, (72\%) e jovens com menos de 40 anos (100\%). Dentre as questões de ansiedade, em sua totalidade (100\%), foram relatados sintomas como insônia, esgotamento e depressão. Conclusão: A pandemia causada pela Covid-19 intensificou diversos fatores causadores de ansiedade em profissionais da saúde, entre eles estão alta carga de trabalho, longas jornadas e estresse. A compreensão desses fatores apresenta-se relevante para a adoção de medidas que possam evitar tais prejuízos para os profissionais de saúde.

Palavras-chave: Ansiedade; Infecções por coronavírus; Saúde mental; Saúde do trabalhador; Equipe de assistência ao paciente.

\footnotetext{
Abstract

Objective: To characterize in the scientific literature studies about anxiety in health professionals working to combat the new coronavirus pandemic and to identify the main physiological and psychological changes among health professionals working in the Covid-19 pandemic. Method: This is an integrative review, and the search was carried out in January 2021, in the following databases: LILACS, MEDLINE and BDENF. The descriptors Anxiety, Coronavirus Infections and Health Personnel were used and the inclusion criteria were complete articles, in Portuguese, English and Spanish, published between 2019 and 2021. It was decided to exclude literature reviews and opinion articles. Results: 50 articles were analyzed. Participants were nurses in 50\% of the articles; doctors (26\%) and health workers $(24 \%)$. Most participants were female (72\%) and younger than 40 years (100\%). Among the anxiety
} 
issues, in its entirety (100\%), symptoms such as insomnia, exhaustion and depression were reported. Conclusion: the pandemic caused by Covid-19 intensified several anxiety-causing factors in health professionals, including high workload, long hours and stress. Understanding these factors is relevant for the adoption of measures that can prevent such damage to health professionals.

Keywords: Anxiety; Coronavirus infections; Mental health; Occupational health; Patient care team.

\section{Resumen}

Objetivo: Caracterizar en la literatura científica los estudios sobre ansiedad en profesionales de la salud que trabajan para combatir la nueva pandemia de coronavirus e identificar los principales cambios fisiológicos y psicológicos entre los profesionales de la salud que trabajan en la pandemia de Covid-19. Método: Se trata de una revisión integradora, y la búsqueda se realizó en enero de 2021, en las siguientes bases de datos: LILACS, MEDLINE y BDENF. Se utilizaron los descriptores Ansiedad, Infecciones por Coronavirus y Personal de Salud y los criterios de inclusión fueron artículos completos, en portugués, inglés y español, publicados entre 2019 y 2021. Se decidió excluir revisiones de literatura y artículos de opinión. Resultados: se analizaron 50 artículos. Los participantes fueron enfermeras en el $50 \%$ de los artículos; médicos $(26 \%)$ y trabajadores de la salud (24\%). La mayoría de los participantes eran mujeres (72\%) y menores de 40 años (100\%). Entre los problemas de ansiedad, en su totalidad $(100 \%)$, se reportaron síntomas como insomnio, agotamiento y depresión. Conclusión: La pandemia causada por Covid-19 intensificó varios factores angustiosos en los profesionales de la salud, entre los que se encuentran la alta carga de trabajo, las largas jornadas y el estrés. Comprender estos factores es relevante para la adopción de medidas que puedan prevenir tal daño a los profesionales de la salud.

Palabras clave: Ansiedad; Infecciones por coronavirus; Salud mental; Salud laboral; Grupo de atención al paciente.

\section{Introdução}

O Brasil é o país com maior número de casos de ansiedade na população mundial, com 9,3\% dos brasileiros adoecidos, segundo dados de 2017. (WHO, 2017). Em 2020, o Ministério da Saúde publicou uma pesquisa sobre o impacto da pandemia na saúde mental da população brasileira, na qual constataram altos índices de transtornos como a ansiedade (86,5\%), o estresse pós-traumático (45,5\%) e a depressão grave a (16\%) na população brasileira, sendo a análise de 17.491 pessoas com idade média de 38,3 anos e maioria do sexo feminino. (Pagno, 2020).

Neste contexto, os profissionais de saúde vivenciam fatores de risco no exercício laboral que podem desencadear sofrimento e transtornos mentais. Corroborando com essa afirmação, um estudo de revisão, identificou alguns fatores de risco para o afastamento laboral por transtornos de ansiedade e depressão entre os profissionais da saúde, a exemplo as altas exigências de trabalho, baixa recompensa salarial, conflitos e tensões com outros profissionais e com o paciente e o desequilíbrio entre trabalho e lazer. (Ribeiro, Santos, Silva, Medeiro, \& Fernandes, 2019).

Considerando as altas exigências no trabalho de profissionais da saúde durante a pandemia da Covid-19, verifica-se aumento da exposição a fatores de risco, como longas jornadas de trabalho, decisões difíceis; alta taxa de mortalidade dos pacientes acometidos pela Covid-19 e medo de adoecimento e transmissão da doença para familiares dos profissionais de saúde, devido ao contato direto dos profissionais com pacientes em fase de transmissão. Impactos individuais da pandemia incluem o distanciamento e isolamento social, medo da infeção no ambiente de trabalho, medo da morte e da perda de conhecidos e familiares. (United Nations, 2020).

Diante desse cenário, no qual o ambiente laboral e as condições de trabalho são precárias, a saúde mental dos profissionais de saúde pode sofrer danos como ansiedade, estresse, depressão, Síndrome de Burnout e outros transtornos que são resultantes do esgotamento físico e mental. (Ornell, Halpern, Kessler, \& Narvaez, 2020). Assim, este estudo teve como objetivos caracterizar na literatura científica estudos sobre a ansiedade em profissionais de saúde atuantes no combate a pandemia do novo coronavírus e identificar as principais alterações fisiológicas e psicológicas entre os profissionais da saúde atuantes na pandemia da Covid-19. 


\section{Metodologia}

Trata-se de uma revisão integrativa de literatura (Souza, Silva, \& Carvalho, 2010), na qual seguiu-se os passos propostos por Whittemore e Knafl: 1) problema de pesquisa, 2) levantamento bibliográfico, 3) avaliação e categorização dos dados, 4) análise dos resultados da amostra, 5) apresentação final da revisão e síntese do conhecimento. (Whittemore \& Knafl, 2005).

A questão norteadora foi desenvolvida por meio da estratégia de pesquisa não clínica, caracterizada pelo acrônimo de palavras: População/Paciente/Problema, Interesse e Contexto(Sousa et al., 2018), sendo P: profissionais da saúde, I: ansiedade, Co: pandemia da Covid-19, o que resultou em: "Qual a caracterização dos profissionais da saúde atuantes durante a pandemia da Covid-19 que apresentaram ansiedade?” e “Quais as principais alterações fisiológicas e psicológicas identificadas entre os profissionais da saúde atuantes na pandemia da Covid-19?”

A pesquisa dos textos foi realizada em janeiro de 2021, nos meios eletrônicos: Literatura LatinoAmericana e do Caribe em Ciências e Saúde (LILACS), Medical Literature Analysis and Retrieval System Online (MEDLINE/PubMed) e BDENF (Banco de dados Bibliográficos Especializado na Área de Enfermagem do Brasil), que estavam contidas no Portal Regional da Biblioteca Virtual em Saúde (BVS).

Para tanto, optou-se em utilizar os descritores em inglês: Anxiety, Coronavirus Infections, Health Personnel; controlados pelos Descritores em Ciências da Saúde (DeCS) e Medical Subject Headings (MeSH). Eles foram selecionados mediante sua relevância e abrangência para o tema e análise de seus conceitos. A busca foi refinada através do operador booleano AND, permitindo a seguinte combinação dos descritores: Anxiety AND Coronavirus Infections AND Health Personnel.

Os critérios utilizados para a inclusão foram: artigos disponíveis completos, idioma (português, inglês e espanhol) e recorte temporal (anos 2019 a 2021), justifica-se esse período devido o ano de início da pandemia e até a data que foi realizada a coleta de dados da pesquisa. Como critérios de exclusão, optou-se por: revisões de literatura, editoriais, artigos de opinião ou guias de prática clínica, estudos que não tivessem como participantes profissionais da saúde, estudos que não tinham a ansiedade descrita e aqueles repetidos nas bases de dados foram considerados uma única vez.

Na primeira busca foram encontrados 250 artigos conforme os critérios de inclusão e com a inserção dos critérios de exclusão, selecionados no site da BVS, a amostra resultou em 197 artigos científicos. Desses, 190 estavam indexados na MEDLINE, 5 LILACS e 2 BDENF. Por último, realizou-se a leitura analítica do título e resumo de cada artigo, excluindo as revisões de literatura, os artigos de opinião e os estudos fora do tema, o que resultou em 50 artigos para essa revisão. O processo de seleção dos estudos baseou-se nas recomendações do Preferred Reporting Items for Systematic Reviews and MetaAnalyses (PRISMA), ilustrado na Figura 1. 
Figura 1 - Fluxograma da seleção dos estudos, adaptado do modelo PRISMA. Curitiba, PR, Brasil, 2021. (Moher, Liberati, Tetzlaff, Altman, \& The PRISMA Group, 2009).

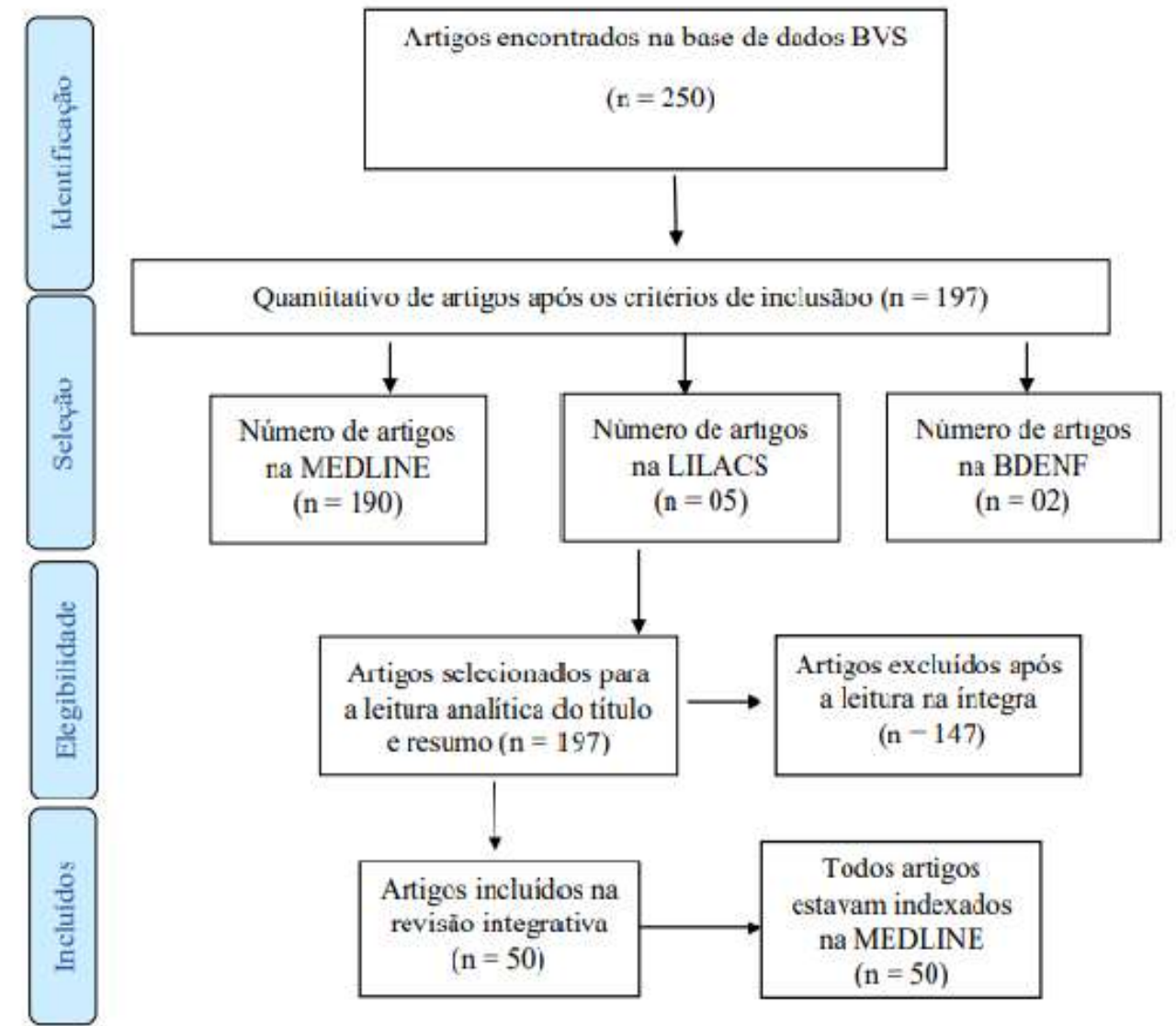

Fonte: Autores (2021).

\section{Resultados}

A amostra final foi composta por 50 artigos, sendo 26,32\% de estudos científicos sobre ansiedade presente em profissionais da saúde atuantes durante a pandemia de Covid-19. Todos estavam disponíveis na MEDLINE e no idioma inglês, apenas um estava publicado no idioma português. Todos os artigos científicos foram publicados no ano de 2020 , o que se justifica pelo fato da pandemia ter sido intensa em todos os países neste ano.

Os estudos foram desenvolvidos em 23 países, dentre eles: China com 15 (30\%), Turquia com cinco (10\%), seguido dos Estados Unidos com quatro (8\%), Itália com três (6\%), Arábia Saudita, Brasil, Espanha, Nepal e França com dois (4\%) respectivamente, assim como Israel, Japão, Irã, Noruega, Egito, Jerusalém, Coreia do Sul, Lêmen, Paquistão, Portugal, Peru, Croácia e Polônia com um (2\%) artigo cada.

Com relação à população incluída nos artigos, observou-se que em 36 (72\%) deles, houve a prevalência de participantes do sexo feminino. Quanto à idade, na totalidade dos 50 estudos (100\%), eram jovens com menos de 40 anos. Referente a categoria profissional, observou-se a predominância de enfermeiros em 25 (50\%) dos estudos, na sequência os médicos em 13 (26\%) e por fim, em 12 (24\%) os trabalhadores de saúde no geral. O tempo de formação mencionado nos estudos foi de pelo menos 10 anos de experiência profissional.

Dentre as questões de ansiedade identificadas nos profissionais de saúde, em sua totalidade nos 50 estudos (100\%), foram relatados sintomas como insônia, esgotamento, a própria ansiedade e depressão. A Tabela 1 apresenta as principais características dos artigos que foram selecionados para essa revisão. 
Tabela 1 - Caracterização dos artigos selecionados. Curitiba, PR, Brasil, 2021.

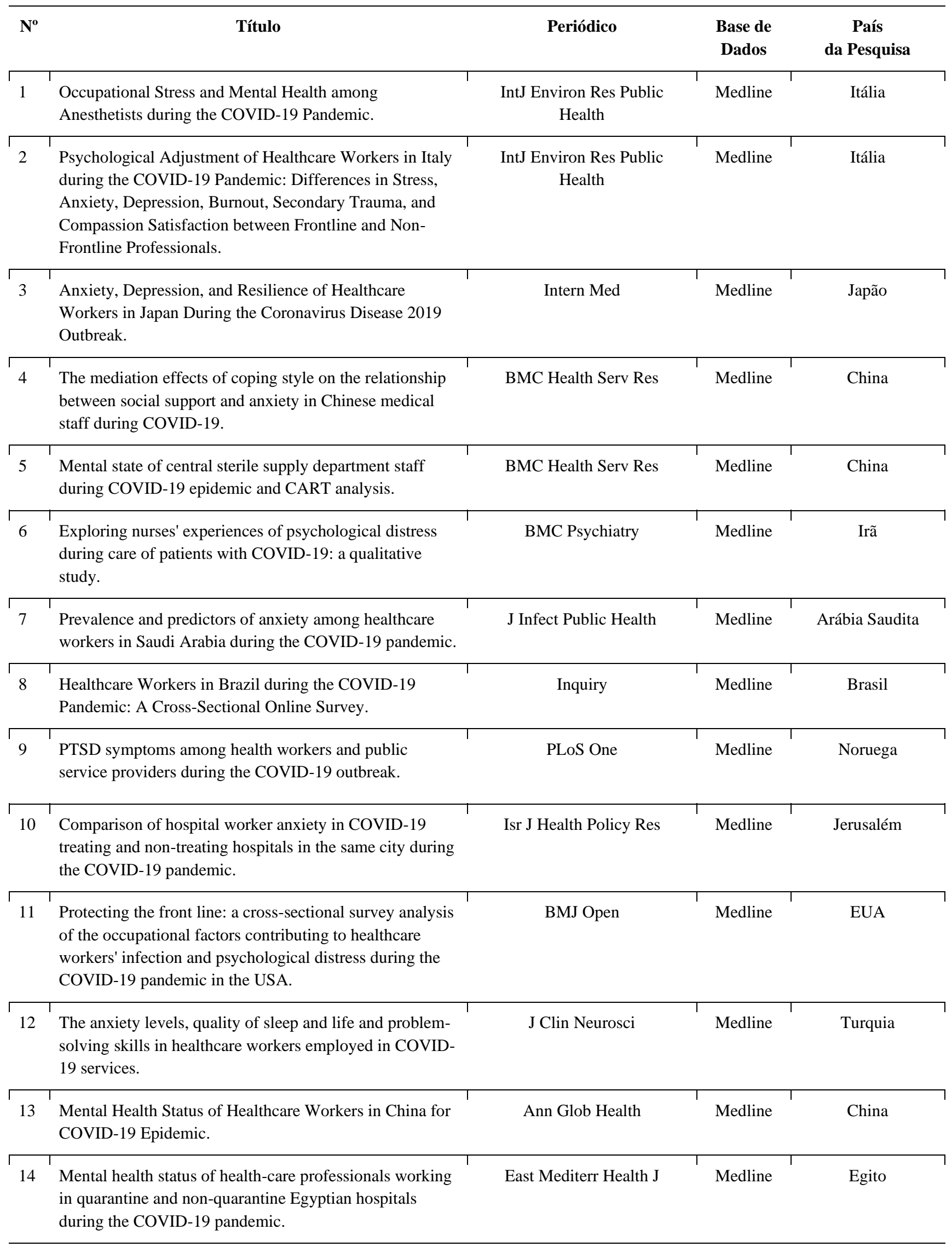


15 A hybrid inductive-abductive analysis of health workers' experiences and wellbeing during the COVID-19

PLoS One

Medline

EUA pandemic in the United States.

\begin{tabular}{|l|l|l|l|l|}
\hline 16 & $\begin{array}{l}\text { COVID-19 Outbreak and Its Association with Healthcare } \\
\text { Workers' Emotional Stress: a Cross-Sectional Study. }\end{array}$ & J Korean Med Sci & Medline & $\begin{array}{l}\text { Coreia } \\
\text { do Sul }\end{array}$ \\
\hline 17 & Knowledge, attitudes, anxiety, and preventive behaviours & BMC Public Health & Medline & Lêmen \\
\hline
\end{tabular}
towards COVID-19 among health care providers in Yemen: an online cross-sectional survey.

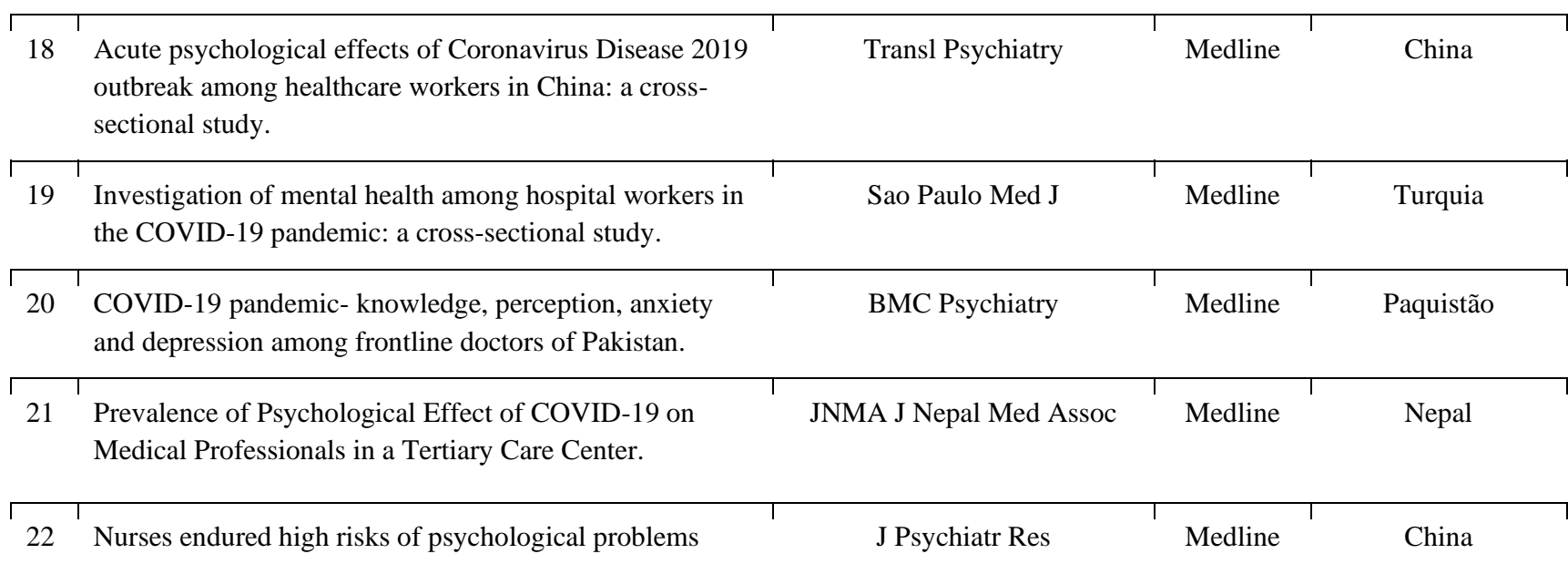
under the epidemic of COVID-19 in a longitudinal study in Wuhan China.

\begin{tabular}{l|l|l|l|}
\hline 23 & $\begin{array}{l}\text { Mental health impacts among health workers during } \\
\text { COVID-19 in a low resource setting: a cross-sectional } \\
\text { survey from Nepal. }\end{array}$ \\
\hline 24 & $\begin{array}{l}\text { The psychological status of } 8817 \text { hospital workers during } \\
\text { COVID-19 Epidemic: A cross-sectional study in } \\
\text { Chongqing. }\end{array}$ \\
\hline 25 & The mental health of neurological doctors and nurses in & BMC Psychiatry & Medline
\end{tabular}
Hunan Province, China during the initial stages of the COVID-19 outbreak.

\begin{tabular}{|l|l|l|l|}
\hline 26 & $\begin{array}{l}\text { Impacts of the Coronavirus Disease 2019 (COVID-19) } \\
\text { pandemic on healthcare workers: A nationwide survey of } \\
\text { United States radiologists. }\end{array}$ \\
\hline 27 & $\begin{array}{l}\text { The Psychological Status and Self-Efficacy of Nurses } \\
\text { During COVID-19 Outbreak: A Cross-Sectional Survey. }\end{array}$ \\
\hline 28 & $\begin{array}{l}\text { Prevalence of Depression, Anxiety, Distress and } \\
\text { Insomnia and Related Factors in Healthcare Workers } \\
\text { During COVID-19 Pandemic in Turkey. }\end{array}$ \\
\hline 29 & $\begin{array}{l}\text { Mental Health in Frontline Medical Workers during the } \\
\text { 2019 Novel Coronavirus Disease Epidemic in China: A } \\
\text { Comparison with the General Population. }\end{array}$ \\
\hline 30 & $\begin{array}{l}\text { Association of Social Network Use With Increased } \\
\text { Anxiety Related to the COVID-19 Pandemic in } \\
\text { Anesthesiology, Intensive Care, and Emergency Medicine }\end{array}$ \\
\hline
\end{tabular}


Teams: Cross-Sectional Web-Based Survey Study.

\begin{tabular}{|l|l|l|l|l|}
\hline 31 & $\begin{array}{l}\text { Mental health among healthcare providers during } \\
\text { coronavirus disease (COVID-19) outbreak in Saudi } \\
\text { Arabia. }\end{array}$ & J Infect Public Health & Medline & Arábia Saudita \\
\hline 32 & $\begin{array}{l}\text { Symptoms of Anxiety, Depression, and Peritraumatic } \\
\text { Dissociation in Critical Care Clinicians Managing } \\
\text { Patients with COVID-19. A Cross-Sectional Study. }\end{array}$ & Am J Respir Crit Care Med & Medline & França \\
& & & \\
\hline
\end{tabular}

\begin{tabular}{l|l|c|c|c|}
\hline 33 & $\begin{array}{l}\text { Nurses' Mental Health During the Covid-19 Outbreak: A } \\
\text { Cross-Sectional Study. }\end{array}$ \\
\hline 34 & Anxiety, Distress, and Turnover Intention of Healthcare & Am J Trop Med Hyg & Medline & Portugal \\
\hline
\end{tabular}
Workers in Peru by Their Distance to the Epicenter during the COVID-19 Crisis.

\begin{tabular}{|l|l|c|c|c|}
\hline 35 & $\begin{array}{l}\text { Symptoms of Posttraumatic Stress, Anxiety, Depression, } \\
\text { Levels of Resilience and Burnout in Spanish Health } \\
\text { Personnel during the COVID-19 Pandemic. }\end{array}$ & $\begin{array}{c}\text { Int J Environ Res Public } \\
\text { Health }\end{array}$ & Medline & Espanha \\
\hline 36 & $\begin{array}{l}\text { Anxiety and hopelessness levels in COVID-19 pandemic: } \\
\text { A comparative study of healthcare professionals and other } \\
\text { community sample in Turkey. }\end{array}$ & J Psychiatr Res & Medline & Turquia \\
\hline 37 & \begin{tabular}{l} 
Psychological impact of coronavirus disease (2019) \\
\hline
\end{tabular} & J Psychiatr Res & Medline & China
\end{tabular}
(COVID-19) epidemic on medical staff in different posts in China: A multicenter study.

\begin{tabular}{|c|c|c|c|c|c|c|}
\hline 38 & $\begin{array}{l}\text { Psychological impact of COVID-19 on medical care } \\
\text { workers in China. }\end{array}$ & 1 & Infect Dis Poverty & & Medline & China \\
\hline 39 & $\begin{array}{l}\text { Anxiety About the Risk of Death of Their Patients in } \\
\text { Health Professionals in Spain: Analysis at the Peak of the } \\
\text { COVID-19 Pandemic. }\end{array}$ & $T$ & $\begin{array}{l}\text { Int J Environ Res Public } \\
\text { Health }\end{array}$ & $\mathrm{T}$ & Medline & Espanha \\
\hline 40 & $\begin{array}{l}\text { Differences in Distress and Coping with the COVID-19 } \\
\text { Stressor in Nurses and Physicians. }\end{array}$ & T & Psychiatr Danub & $\mathrm{T}$ & Medline & Croácia \\
\hline 41 & $\begin{array}{l}\text { Assessment of Mental Health Factors among Health } \\
\text { Professionals Depending on Their Contact with COVID- } \\
19 \text { Patients. }\end{array}$ & & $\begin{array}{l}\text { Int J Environ Res Public } \\
\text { Health }\end{array}$ & & Medline & Polônia \\
\hline 42 & $\begin{array}{l}\text { Anxiety, pandemic-related stress and resilience among } \\
\text { physicians during the COVID-19 pandemic. }\end{array}$ & $T$ & Depress Anxiety & $\mathrm{T}$ & Medline & Israel \\
\hline 43 & $\begin{array}{l}\text { The mental health of frontline and non-frontline medical } \\
\text { workers during the coronavirus disease } 2019 \text { (COVID- } \\
\text { 19) outbreak in China: A case-control study. }\end{array}$ & $T$ & J Affect Disord & $T$ & Medline & China \\
\hline 44 & $\begin{array}{l}\text { Psychological impact of healthcare workers in China } \\
\text { during COVID-19 pneumonia epidemic: A multi-center } \\
\text { cross-sectional survey investigation. }\end{array}$ & & J Affect Disord & & Medline & China \\
\hline 45 & $\begin{array}{l}\text { Mental health of nursing in coping with COVID-19 at a } \\
\text { regional university hospital. }\end{array}$ & & Rev Bras Enferm & & Medline & Brasil \\
\hline
\end{tabular}




\begin{tabular}{l}
\hline $46 \begin{array}{l}\text { Symptoms in Health Care Workers during the COVID-19 } \\
\text { Epidemic. A Cross-Sectional Survey. }\end{array}$ Int J Environ Res Public \\
Health
\end{tabular}

Fonte: Autores (2021).

\section{Discussão}

A situação crítica dos serviços de saúde, desencadeada pela pandemia, se reflete diretamente na saúde mental dos profissionais de saúde. (Liu et al., 2020; Alrubaiee, Al-Qalah, \& Al-Aawar, 2020). Nesta revisão, os estudos selecionados demonstram a prevalência de alterações psicológicas, tais como ansiedade, entre os profissionais da saúde atuantes na linha de frente do combate a Covid-19. (Wang et. al., 2020).

Neste contexto, problemas relacionados à ansiedade estão cada vez mais constantes no mundo. Destaca-se que a maioria dos estudos selecionados foram realizados na China, região do epicentro da disseminação do SARS-CoV-2. (Felício, Rossi, \& Pereira, 2020). Portanto, foi o primeiro país a enfrentar este grande desafio, e observar seus reflexos na saúde dos profissionais de saúde. (Xiaoming et al., 2020; Santos et al., 2020; Liang et al., 2020).

A maioria dos participantes incluídos nos estudos, eram do sexo feminino (Araç, \& Dönmezdil, 2020; Ning et al., 2020; Lázaro-Pérez, Martínez-López, Gómez-Galán, \& López-Meneses, 2020), o que se justifica pela questão de que as mulheres são a maioria na área de saúde. (Santos, Lima, Barbosa, Silva, \& Andrade, 2020). Em alguns estudos houve o predomínio do sexo masculino, principalmente naqueles cuja população era formada exclusivamente por médicos. (Korkmaz et al., 2020; Youssef, Mostafa, Ezzat, Yosef, \& Kassas, 2020).

Ressalta-se que a população era de adultos jovens (Zhu, Wei, Meng, \& Li, 2020; Cotrin et al., 2020; Youssef, Mostafa, Ezzat, Yosef, \& Kassas, 2020) e com cerca de 10 anos de formação profissional (Park, Hwang, Jo, Bae, \& Sakong, 2020; AlAteeq, Aljhani, Althiyabi, \& Majzoub, 2020). Em se tratando de ocupação, a maioria trata-se de enfermeiros (Pan, Hu, \& Yi, 2020; Alrubaiee, Al-Qalah, \& Al-Aawar, 2020), os quais representam a maior classe de trabalhadores da saúde. (Vieira et al., 2021). Segundo dados da Organização Mundial da Saúde (OMS), há aproximadamente 28 milhões de enfermeiros mundialmente (OPAS Brasil, 2020), sendo a classe ocupacional com a maior força de trabalho na área da saúde. Entretanto, a profissão ainda necessita de reconhecimento da população e dos governantes e ainda luta por melhores salários e condições de trabalho.

Entre as principais alterações fisiológicas e psicológicas identificadas e que acometeram os profissionais de saúde, destaca-se a prevalências de insônia, esgotamento, estresse, ansiedade e até mesmo a depressão. (Trumello, et al., 2020; Luceño-Moreno, Talavera-Velasco, García-Albuerne, \& Martín-García, 2020; Magnavita, Tripepi, \& Di Prinzio, 2020).O desenvolvimento destas alterações psicológicas correspondem ao estresse relacionado pelo desgaste ocasionado pela 
sobrecarga laboral, às condições precárias de trabalho, ao sentimento de impotência e ao número elevado de óbitos. (Galehdar, Kamran, Toulabi, \& Heydari, 2020; Johnson, Ebrahimi, \& Hoffart, 2020; Magnavita, Tripepi, \& Di Prinzio, 2020).

Outra questão observada foi o medo de adoecer e de infectar membros da família (Araç, \& Dönmezdil, 2020).Isso demonstra a importância da implementação de políticas trabalhistas e de saúde para estes profissionais, em contrapartida a sociedade também precisa fazer sua parte e cumprir as medidas para controle da pandemia.

Um fator preocupante é que dentre os profissionais de saúde, o estado psicológico dos enfermeiros requer um pouco mais de atenção. (Cai et al., 2020). Identificou-se que eles estão entre os profissionais com maior probabilidade de desenvolver sintomas depressivos (Nobuyasu, et al., 2020; Alenazi, 2020; Xiaoming et al., 2020), o que impacta diretamente em sua qualidade de vida. (Moreira, \& Lucca, 2020). Já na China, foi apontado que a equipe médica apresentou um maior nível de ansiedade durante a pandemia. (Zhu, Wei, Meng, \& Li, 2020).

Também entre os achados, observou-se que há mais ansiedade entre as mulheres do que entre os homens (Araç, \& Dönmezdil, 2020; Ning et al., 2020). Infere-se com esses resultados que as mulheres apresentam uma responsabilidade social elevada, visto que na maioria das sociedades elas são responsáveis pelo cuidado de seus filhos e familiares.

Por fim, os estudos evidenciam a importância da oferta de suporte psicológico aos profissionais de saúde, a fim de minimizar os riscos para a saúde mental ocasionados pelo contexto da pandemia. (Nobuyasu, et al., 2020; Liu et al., 2020). Ressalta-se a necessidade de investimentos das políticas públicas de saúde mental destinadas à prevenção do adoecimento desses profissionais.

O estudo apresenta como limitação o número de bases pesquisadas, bem como apresenta lacunas de conhecimento que poderiam ter sido discutidas como ações em saúde efetivas que vêm sendo utilizadas para tratar desses profissionais da saúde. Também, percebe-se como fragilidade a falta de uma gestão de saúde pública nos países, demonstrando que há necessidade de investimentos em políticas públicas que permitam assistir os profissionais de saúde durante e pós-pandemia a fim de evitar o sofrimento mental e psíquico.

\section{Conclusão}

A compreensão sobre fatores de adoecimento mental em profissionais de saúde causados pela pandemia apresenta-se relevante para a adoção de medidas que possam evitar tais prejuízos à saúde mental deles, evitando absenteísmo, afastamentos prolongados, sofrimento psíquico e incapacidades. Estratégias de suporte emocional, melhorias nas condições de trabalho e prevenção do adoecimento mental podem ser utilizadas para amenizar os impactos da pandemia da Covid-19 nestes profissionais. Recomenda-se que novos estudos analisem a presença de outros fatores de adoecimento mental, como estresse, depressão, burnout.

\section{Referências}

AlAteeq, D. A., Aljhani, S., Althiyabi, I., \& Majzoub, S. (2020). Mental health among healthcare providers during coronavirus disease (COVID-19) outbreak in Saudi Arabia. Journal of Infection and Public Health, 13(10), 1432-1437. https://www.sciencedirect.com/science/article/pii/ S1876034120306353?via\%3Dihub https://doi.org/10.1016/j.jiph.2020.08.013

Alenazi, T. H., BinDhim, N. F., Alenazi, M. H., Tamim, H., Almagrabi, R. S., Aljohani, S. M., Basyouni, M. H., Almubark, R. A., Althumiri, N. A., \& Alqathani, S. A. (2020). Prevalence and predictors of anxiety among healthcare workers in Saudi Arabia during the COVID-19 pandemic. Journal of Infection and Public Health, 13, $1645-51$ https://reader.elsevier.com/reader/sd/pii/S1876034120306377?token=8C2051BC2 6A4E03636D2E9BB9AA0E009F3657938E5F2271130C4BCFBF9EE6ECF95407B8DFB3A182C7EEA3DEB4DA14EA2\&originRegion=us-east-

1\&originCreation=20210703180813 https://doi.org/10.1016/j.jiph.2020.09.001

Alrubaiee, G. G., Al-Qalah, T. A. H., \& Al-Aawar, M. S. A. (2020). Knowledge, attitudes, anxiety, and preventive behaviours towards COVID-19 among health care providers in Yemen: an online cross-sectional survey. BMC Public Health, 20(1), 1541. https://bmcpublichealth.biomedcentral.com/track/pdf/10.1186/s12889-020-09644-y.pdf https://doi.org/10.1186/s12889-020-09644-y 
Amin, F., Sharif, S., Saeed, R., Durrani, N., \& Jilani, D. (2020). COVID-19 pandemic- knowledge, perception, anxiety and depression among frontline doctors of Pakistan. BMC Psychiatry, 20 (459). https://bmcpsychiatry.biomedcentral.com/track/pdf/10.1186/s12888-020-02864-x.pdf https://doi.org/10.1186/s12888020-02864-x

Araç, S., \& Dönmezdil, S. (2020). Investigation of mental health among hospital workers in the COVID-19 pandemic: a cross-sectional study. São Paulo Medical Journal, 138(5), 433-440. https://www.scielo.br/j/spmj/a/rJXBWTHvywNTnBCQrwrQTcG/?lang=en https://doi.org/10.1590/15163180.2020.0272.R3.21072020

Azoulay E, Cariou A, Bruneel F, Demoule A, Kouatchet A, Reuter D, Souppart, V., Combes, A., Klouche, K., Argaud, L., Barbier, F., Jourdain, M., Reignier, J., Papazian, L., Guidet, B., Géri, G., Resche-Rigon, M., Guisset, O., Labbé, V., Mégarbane, B., Meersch, G. V. D., Guitton, C., Friedman, D., Pochard, F., Darmon, M., Kentish-Barnes, N. (2020). Symptoms of Anxiety, Depression, and Peritraumatic Dissociation in Critical Care Clinicians Managing Patients with COVID-19. A Cross-Sectional Study. American Journal of Respiratory and Critical Care Medicine, 202(10), 1388-1398. https://www.atsjournals.org/doi/10.1164/rccm.202006-2568OC https://doi.org/10.1164/rccm.202006-2568OC

Cai, Q., Feng, H., Huang, J., Wang, M., Wang, Q., Lu, X., Xie, Y., Wang, X., Liu, Z., Hou, B., Ouyang, K., Pan, J., Li, Q., Fu, B., Deng, Y., \& Liu, Y. (2020). The mental health of frontline and non-frontline medical workers during the coronavirus disease 2019 (COVID-19) outbreak in China: A case-control study. Journal of Affective Disorders, 275, 210-215. https://www.sciencedirect.com/science/article/abs/pii/S0165032720323892?via\%3Dihub https://doi.org/10.1016/j.jad.2020.06.031

Cai, Z., Cui, Q., Liu, Z., Li, J., Gong, X., Liu, J., Wan, Z., Yuan, X., Li, X., Chen, C., \& Wang, G. (2020). Nurses endured high risks of psychological problems under the epidemic of COVID-19 in a longitudinal study in Wuhan China. Journal of Psychiatric Research, 131, 132-137. https://www.sciencedirect.com/science/article/abs/pii/S0022395620309651?via\%3Dihub https://doi.org/10.1016/j.jpsychires.2020.09.007

Clavier, T., Popoff, B., Selim, J., Beuzelin, M., Roussel, M., Compere, V., Veber, B., \& Besnier, E. (2020). Association of Social Network Use With Increased Anxiety Related to the COVID-19 Pandemic in Anesthesiology, Intensive Care, and Emergency Medicine Teams: Cross-Sectional Web-Based Survey Study. JMIR Mhealth Uhealth, 8(9), 1-10. https://mhealth.jmir.org/2020/9/e23153/ https://dx.doi.org/10.2196\%2F23153

Cotrin, P., Moura, W., Gambardela-Tkacz, C. M., Pelloso, F. C., Santos, L. D., Carvalho, M. D. B., Pelloso, S. M., \& Freitas, K. M. S. (2020). Healthcare Workers in Brazil during the COVID-19 Pandemic: A Cross-Sectional Online Survey. Inquiry, 57, 1-11. https://journals.sagepub.com/doi/pdf/10.1177/0046958020963711 https://doi.org/10.1177\%2F0046958020963711

Dal'Bosco, E. B., Floriano, L. S. M., Skupien, S. V., Arcaro, G., Martins, A. R., \& Anselmo, A. C. (2020). Mental health of nursing in coping with COVID-19 at a regional university hospital. Revista Brasileira de Enfermagem, 73 (suppl 2) 1-7. https://www.scielo.br/j/reben/a/ck98YrXKhsh6mhZ3RdB8ZVX/?format=pdf\&lang=pt https://doi.org/10.1590/0034-7167-2020-0434

Demirjian, N. L., Fields, B. K. K., Song, C., Reddy, S., Desai, B., Cen, S. Y., Salehi, S., \& Gholamrezanezhad, A. (2020). Impacts of the Coronavirus Disease 2019 (COVID-19) pandemic on healthcare workers: A nationwide survey of United States radiologists. Clinical Imaging, 68, 218-225. https://www.clinicalimaging.org/action/showPdf?pii=S0899-7071\%2820\%2930330-2 https://doi.org/10.1016/j.clinimag.2020.08.027

Felício, L. V., Rossi, C. C., \& Pereira, M. F. (2020). The emergence of a new zoonotic coronavirus: SARS-Cov-2 and the COVID-19 pandemic. Sapiens. 2(2), 21-38. https://revista.uemg.br/index.php/sps/article/download/5271/3311

Firew, T., Sano, E. D., Lee, J. W., Flores, S., Lang, K., Salman, K., Greene, M. C., \& Chang, B. P. (2020). Protecting the front line: a cross-sectional survey analysis of the occupational factors contributing to healthcare workers' infection and psychological distress during the COVID-19 pandemic in the USA. BMJ Open, 10(10), 1-12. https://bmjopen.bmj.com/content/bmjopen/10/10/e042752.full.pdf https://doi.org/10.1136/bmjopen-2020-042752

Galehdar, N., Kamran, A., Toulabi, T., \& Heydari, H. (2020). Exploring nurses' experiences of psychological distress during care of patients with COVID-19: a qualitative study. BMC Psychiatry; 20(489). https://bmcpsychiatry.biomedcentral.com/track/pdf/10.1186/s12888-020-02898-1.pdf https://doi.org/10.1186/s12888-020-02898-1

Hacimusalar, Y., Kahve, A. C., Yasar, A. B., \& Aydin, M. S. (2020). Anxiety and hopelessness levels in COVID-19 pandemic: A comparative study of healthcare professionals and other community sample in Turkey. Journal of Psychiatric Research, 129, 181-188. https://pubmed.ncbi.nlm.nih.gov/32758711/ https://doi.org/10.1016/j.jpsychires.2020.07.024

Hennein, R., \& Lowe, S. (2020). A hybrid inductive-abductive analysis of health workers' experiences and wellbeing during the COVID-19 pandemic in the United States. PLoS Medicine, 15(10), 1-21. https://journals.plos.org/plosone/article?id=10.1371/journal.pone.0240646 https://doi.org/10.1371/journal.pone.0240646

Johnson, S. U., Ebrahimi, O. V., \& Hoffart, A. (2020). PTSD symptoms among health workers and public service providers during the COVID-19 outbreak. PLOS ONE, 15(10), 1-13. https://journals.plos.org/plosone/article?id=10.1371/journal.pone.0241032 https://dx.doi.org/10.1371\%2Fjournal.pone.0241032

Khanal, P., Devkota, N., Dahal, M., Paudel, K., \& Joshi, D. (2020). Mental health impacts among health workers during COVID-19 in a low resource setting: a cross-sectional survey from Nepal. Globalization and Health, 16(1), 89. https://globalizationandhealth.biomedcentral.com/track/pdf/10.1186/s12992-02000621-z.pdf https://doi.org/10.1186/s12992-020-00621-z

Korkmaz, S., Kazgan, A., Çekiç, S., Tartar, A. S., Balcı, H. N., \& Atmaca, M. (2020). The anxiety levels, quality of sleep and life and problem-solving skills in healthcare workers employed in COVID-19 services. Journal of Clinical Neuroscience, 80, 131-136. https://www.jocn-journal.com/article/S09675868(20)31439-9/fulltext https://doi.org/10.1016/j.jocn.2020.07.073

Lázaro-Pérez, C., Martínez-López, J. Á., Gómez-Galán, J., \& López-Meneses, E. (2020). Anxiety About the Risk of Death of Their Patients in Health Professionals in Spain: Analysis at the Peak of the COVID-19 Pandemic. International Journal of Environment Research of Public Health, 17(16), 5938. https://www.mdpi.com/1660-4601/17/16/5938 https://doi.org/10.3390/ijerph17165938

Liang, Y., Wu, K., Zhou, Y., Huang, X., Zhou, Y., \& Liu, Z. (2020). Mental Health in Frontline Medical Workers during the 2019 Novel Coronavirus Disease Epidemic in China: A Comparison with the General Population. International Journal of Environment Research of Public Health, 17(18), 6550. https://www.mdpi.com/1660-4601/17/18/6550 https://doi.org/10.3390/ijerph17186550 
Liu, Z., Wu, J., Shi, X., Ma, Y., Ma, X., Teng, Z. You, X., Zhang, Y., Zhang, W., Feng, Z., Long, Q., Ma, X., Wang, L., \& Zeng, Y. (2020). Mental Health Status of Healthcare Workers in China for COVID-19 Epidemic. Annals of Global Health, 86(1): 128, 1-8. https://annalsofglobalhealth.org/articles/10.5334/aogh.3005/ https://dx.doi.org/10.5334\%2Faogh.3005

Lu, W., Wang, H., Lin, Y., \& Li, L. (2020). Psychological status of medical workforce during the COVID-19 pandemic: A cross-sectional study. Psychiatry Research, 288. https://www.sciencedirect.com/science/article/abs/pii/S0165178120305850?via\%3Dihub https://doi.org/10.1016/j.psychres.2020.112936

Luceño-Moreno, L., Talavera-Velasco, B., García-Albuerne, Y., \& Martín-García, J. (2020). Symptoms of Posttraumatic Stress, Anxiety, Depression, Levels of Resilience and Burnout in Spanish Health Personnel during the COVID-19 Pandemic. International Journal of Environment Research and Public Health, 17(15), 5514. https://www.mdpi.com/1660-4601/17/15/5514 https://doi.org/10.3390/ijerph17155514

Magnavita, N., Soave, P. M., Ricciardi, W., \& Antonelli, M. (2020). Occupational Stress and Mental Health among Anesthetists during the COVID-19 Pandemic. International Journal of Environmental Research and Public Health, 17(21), 8245. https://www.mdpi.com/1660-4601/17/21/8245/htm https://dx.doi.org/10.3390\%2Fijerph17218245

Magnavita, N., Tripepi, G., \& Di Prinzio, R. R. (2020). Symptoms in Health Care Workers during the COVID-19 Epidemic. A Cross-Sectional Survey. International Journal of Environment Research of Public Health, 17(14), 5218. https://www.mdpi.com/1660-4601/17/14/5218 https://dx.doi.org/10.3390\%2Fijerph17145218

Milgrom, Y., Tal, Y., \& Finestone, A. S. (2020). Comparison of hospital worker anxiety in COVID-19 treating and non-treating hospitals in the same city during the COVID-19 pandemic. Israel Journal of Health Policy Research, 9(1). https://ijhpr.biomedcentral.com/track/pdf/10.1186/s13584-020-00413-1.pdf https://doi.org/10.1186/s13584-020-00413-1

Moher, D., Liberati, A., Tetzlaff, J., Altman, D. G., \& The PRISMA Group. (2009). Preferred Reporting Items for Systematic Reviews and Meta-Analyses: The PRISMA Statement. PLoS Medicine, 6(7). https://journals.plos.org/plosmedicine/article?id=10.1371/journal.pmed.1000097 https://doi.org/10.1371/journal.pmed.1000097

Moreira, A. S., \& Lucca, S. R. (2020). Psychosocial factors and Burnout Syndrome among mental health professionals. Revista Latino-Americana de Enfermagem, 28. https://www.scielo.br/j/rlae/a/Qvm6b5FzSBCXTLLSsfTpRVd/?format=pdf\&lang=pt https://doi.org/10.1590/1518-8345.4175.3336

Mosheva, M., Hertz-Palmor, N., Ilan, S. D., Matalon, N., Pessach, I. M., Afek, A., Ziv, A., Kreiss, Y., Gross, R., \& Gothelf, D. (2020). Anxiety, pandemicrelated stress and resilience among physicians during the COVID-19 pandemic. Depression and Anxiety, 37(10), 965-971. https://onlinelibrary.wiley.com/doi/10.1002/da.23085 https://doi.org/10.1002/da.23085

Ning, X., Yu, F., Huang, Q., Li, X., Luo, Y., Huang, Q., \& Chen, C. (2020). The mental health of neurological doctors and nurses in Hunan Province, China during the initial stages of the COVID-19 outbreak. BMC Psychiatry, 20(1), 436. https://bmcpsychiatry.biomedcentral.com/track/pdf/10.1186/s12888-02002838-z.pdf https://doi.org/10.1186/s12888-020-02838-z

Nobuyasu, A., Oyama, N., Akiyama, K., Inomata, M., Kuse, N., Tone, M., Takada, K., Muto, Y., Fujimoto, K., Akagi, Y., Mawatari, M., Ueda, A., Kawakami, J., Komatsu, J., \& Izumo, T. (2020). Anxiety, Depression, and Resilience of Healthcare Workers in Japan During the Coronavirus Disease 2019 Outbreak. Internal Medicine, 59(21), 2693-2699. https://www.jstage.jst.go.jp/article/internalmedicine/59/21/59_5694-20/_article https://doi.org/10.2169/internalmedicine.5694-20

Organização Pan Americana de Saúde (OPAS) Brasil. (2020). Dia Mundial da Saúde: OMS e parceiros pedem investimentos urgentes em profissionais de enfermagem. https://www.paho.org/pt/news/7-4-2020-amid-covid-19-pandemic-new-who-report-urges-greater-investments-nursing-workforce

Ornell, F., Halpern, S. C., Kessler, F. H. P., \& Narvaez, J. C. M. (2020). The impact of the COVID-19 pandemic on the mental health of healthcare professionals. Cadernos de Saúde Pública, 36(4). https://www.scielo.br/j/csp/a/w4b7SQrVXtq3DjFbns64pCw/?lang=en https://doi.org/10.1590/0102$311 \times 00063520$

Pagno, M. (2020). Ministério da Saúde divulga resultados preliminares de pesquisa sobre saúde mental na pandemia. https://antigo.saude.gov.br/noticias/agencia-saude/47527-ministerio-da-saude-divulga-resultados-preliminares-de-pesquisa-sobre-saude-mental-na-pandemia

Pan, W., Hu, J., \& Yi, L. (2020). Mental state of central sterile supply department staff during COVID-19 epidemic and CART analysis. BMC Health Services Research, 20(1), 1006. https://bmchealthservres.biomedcentral.com/track/pdf/10.1186/s12913-020-05864-5.pdf https://doi.org/10.1186/s12913-020-05864-5

Park, C., Hwang, J. M., Jo, S., Bae, S. J., \& Sakong, J. (2020). COVID-19 Outbreak and Its Association with Healthcare Workers' Emotional Stress: a CrossSectional Study. Journal of Korean Medical Science, 35(41). https://jkms.org/DOIx.php?id=10.3346/jkms.2020.35.e372 https://dx.doi.org/10.3346\%2Fjkms.2020.35.e372

Ribeiro, H. K. P., Santos J. D. M., Silva M. de G., Medeiro, F. D. de A., \& Fernandes, M. A. (2019). Anxiety disorders as a cause of work absenteeism. Revista Brasileira de Saúde Ocupacional, 44(1). https://www.scielo.br/j/rbso/a/WfpQJQM7TSqLb7PWxW9Frwg/?lang=pt. https://doi.org/10.1590/23176369000021417

Şahin, M. K., Aker, S., Şahin, G., \& Karabekiroğlu, A. (2020). Prevalence of Depression, Anxiety, Distress and Insomnia and Related Factors in Healthcare Workers During COVID-19 Pandemic in Turkey. Journal of Community Health, 45(6), 1168-1177. https://link.springer.com/content/pdf/10.1007/s10900-02000921-w.pdf https://doi.org/10.1007/s10900-020-00921-w

Salopek-Žiha, D., Hlavati, M., Gvozdanović, Z., Gašić, M., Placento, H., Jakić, H., Klapan, D., \&Šimić, H. (2020). Differences in Distress and Coping with the COVID-19 Stressor in Nurses and Physicians. Psychiatria Danubina, 32(2), 287-293. https://pubmed.ncbi.nlm.nih.gov/32796800/ https://doi.org/10.24869/psyd.2020.287

Sampaio, F., Sequeira, C., \& Teixeira, L. (2020). Nurses' Mental Health During the Covid-19 Outbreak: A Cross-Sectional Study. Journal of Occupational and Environmental Medicine, 62(10), 783-787. https://journals.lww.com/joem/Fulltext/2020/10000/Nurses_Mental_Health_During_the_Covid_19.2.aspx https://doi.org/10.1097/jom.0000000000001987 
Santos, G. B. M., Lima, R. C. D., Barbosa, J. P. M., Silva, M. C., \& Andrade, M. A. C. (2020). Self-care: health workers in time of pandemic by COVID-19. Trabalho, Educação e Saúde, 18 (3). https://www.scielo.br/j/tes/a/vS8DgWb8QXTBJkbGnCP4CDQ/?format=pdf\&lang=pt https://doi.org/10.1590/19817746-sol00300

Santos, T. A., Santos, H. S., Sampaio, E. S., Melo, C. M. M., Souza, E. A., \& Pires, C. G. S. (2020). Intensity of nursing work in public hospitals. Revista Latino-Americana de Enfermagem, 28. https://www.scielo.br/j/rlae/a/nRdbkqLnxvvSTTbgRTcRwGt/?format=pdf\&lang=pt https://doi.org/10.1590/15188345.3221 .3267

Shechter, A., Diaz, F., Moise, N., Anstey, D. E., Ye, S., Agarwal, S., Birk, J. L., Brodie, D., Cannone, D. E., Chang, B., Claassen, J., Cornelius, T., Derby, L., Dong, M., Givens, R. C., Hochman, B., Homma, S., Kronish, I. M., Lee, S. A. J., Manzano, W, Mayer, L. E. S., McMurry, C. L., Moitra, V., Pham, P., Rabbani, L. R., Rivera, R. R., Schwartz, A., Schwartz, J. E., Shapiro, P. A., Shaw, K., Sullivan, A. M., Vose, C., Wasson, L., Edmondson, D., \& Abdalla, M. (2020). Psychological distress, coping behaviors, and preferences for support among New York healthcare workers during the COVID-19 pandemic. General Hospital Psychiatry, 66, 1-8. https://www.sciencedirect.com/science/article/abs/pii/S0163834320300839?via\%3Dihub https://doi.org/10.1016/j.genhospp sych.2020.06.007

Shrestha, S. L. (2020). Prevalence of Psychological Effect of COVID-19 on Medical Professionals in a Tertiary Care Center. J Nepal Med Assoc, 58(228), 550-553. http://www.jnma.com.np/jnma/index.php/jnma/article/view/5087/3216 https://doi.org/10.31729/jnma.5087

Si, M. Y., Su, X. Y., Jiang, Y., Wang, W. J., Gu, X. F., Ma, L., Li, J., Zhang, S. K., Ren, Z. F., Ren, R., Liu, Y. L., \& Qiao, Y, L. (2020). Psychological impact of COVID-19 on medical care workers in China. Infectious Diseases of Poverty, 9(1), 113. https://idpjournal.biomedcentral.com/track/pdf/10.1186/s40249020-00724-0.pdf https://doi.org/10.1186/s40249-020-00724-0

Sousa, L. M. M., Marques, J. M., Firmino, C. F., Frade, F., Valentim, O. S., \& Antunes, A. V. (2018). Frameworks to research question in evidence-based practice. Revista Investigação em Enfermagem, S2(23), 31-39. Retirado de https://repositorio-cientifico.essatla.pt/bitstream/20.500.12253/1287/1/artigo3139.pdf

Souza, M. T., Silva, M. D., \& Carvalho, R. (2010). Integrative review: what is it? How to do it?.Eistein, 8(1), 102-6. https://www.scielo.br/j/eins/a/ZQTBkVJZqcWrTT34cXLjtBx/?lang=en http://dx.doi.org/10.1590/s1679-45082010rw1134

Trumello, C., Bramanti, S. M., Ballarotto, G., Candelori, C., Cerniglia, L., Cimino, S., Crudele, M., Lombardi, L., Pignataro, S., Viceconti, M. L., \& Babore, A. (2020). Psychological Adjustment of Healthcare Workers in Italy during the COVID-19 Pandemic: Differences in Stress, Anxiety, Depression, Burnout, Secondary Trauma, and Compassion Satisfaction between Frontline and Non-Frontline Professionals. International Journal of Environmental Research and Public Health, 17(22), 8358. Retirado de https://www.mdpi.com/1660-4601/17/22/8358/htm https://doi.org/10.3390/ijerph17228358

United Nations. (2020). Policy Brief: COVID-19 and the need for action on mental health. https://www.un.org/sites/un2.un.org/files/un_policy_briefcovid_and_mental_health_final.pdf

Vieira, L. J. E. S., Silva, R. M., Filho, C. G., Bezerra, I. C., Júnior, A. R. F., \& Guimarães, J. M. X. (2021). Nursing work: analysis of wage trends in Brazil. Research, Society and Development, 10(3). https://rsdjournal.org/index.php/rsd/article/view/13569 http://dx.doi.org/10.33448/rsd-v10i3.13569

Wang, L. Q., Zhang, M., Liu, G. M., Nan, S. Y., Li, T., Xu, L., Xue, Y., Zhang, M., Wang, L., Qu, Y. D., \& Liu, F. (2020). Psychological impact of coronavirus disease (2019) (COVID-19) epidemic on medical staff in different posts in China: A multicenter study. Journal of Psychiatric Research, 129, 198205. https://www.sciencedirect.com/science/article/abs/pii/S0022395620308724?via\%3Dihub https://doi.org/10.1016/j.jpsychires.2020.07.008

Wang, Y., Ma, S., Yang, C., Cai, Z., Hu, S., Zhang, B. Tang, S., Bai, H., Guo, X., Wu, J., Du, H., Kang, L., Tan, H., Li, R., Yao, L., Wang, G., \& Liu, Z. (2020). Acute psychological effects of Coronavirus Disease 2019 outbreak among healthcare workers in China: a cross-sectional study. Translational Psychiatry, 10(348). https://www.nature.com/articles/s41398-020-01031-w.pdf https://doi.org/10.1038/s41398-020-01031-w

Wańkowicz, P., Szylińska, A., \& Rotter, I. (2020). Assessment of Mental Health Factors among Health Professionals Depending on Their Contact with COVID-19 Patients. International Journal of Environment Research of Public Health, 17(16), 5849. https://www.mdpi.com/1660-4601/17/16/5849 https://doi.org/10.3390/ijerph17165849

Whittemore, R., \& Knafl, K. (2005).The integrative review: updated methodology. J Adv Nurs, 52(5), 546-53. https://onlinelibrary.wiley.com/doi/abs/10.1111/j.1365-2648.2005.03621.x https://doi.org/10.1111/j.1365-2648.2005.03621.x

World Health Organization. (2017). Depression and Other Common Mental Disorders: Global Health Estimates. http://apps.who.int/iris/bitstream/handle/10665/254610/WHO-MSD-MER-2017.2-eng.pdf;jsessionid=B29E12B35C6C182C410A25A92968D543?sequence=1 Geneva.

Xiao, X., Zhu, X., Fu, S., Hu, Y., Li, X., \& Xiao, J. (2020). Psychological impact of healthcare workers in China during COVID-19 pneumonia epidemic: A multi-center cross-sectional survey investigation. Journal of Affective Disorders, 274, 405-410. https://pubmed.ncbi.nlm.nih.gov/32663970/ https://doi.org/10.1016/j.jad.2020.05.081

Xiaoming, X., Ming, A., Su, H., Wo, W., Jianmei, C., Qi, Z., Hua, H., Xuemei, L., Lixia, W., Jun, C., Lei, S., Zhen, L., Lian, D., Jing, L., Handan, Y., Haitang, Q., Xiaoting, H., Xiaorong, C., Ran, C., Qinghua, L., Xinyu, Z., Jian, T., Jing, T., Guanghua, J., Zhigin, H., Nkundimana, B., \& Li, K. (2020). The psychological status of 8817 hospital workers during COVID-19 Epidemic: A cross-sectional study in Chongqing. Journal of Affective Disorders, 276, 555561. https://www.sciencedirect.com/science/article/abs/pii/S0165032720325374?via\%3Dihub https://doi.org/10.1016/j.jad.2020.07.092

Xiong, H., Yi, S., \& Lin, Y. (2020). The Psychological Status and Self-Efficacy of Nurses During COVID-19 Outbreak: A Cross-Sectional Survey. Inquiry, 57, 1-6. https://journals.sagepub.com/doi/pdf/10.1177/0046958020957114 https://doi.org/10.1177/0046958020957114

Yáñez, J. A., Jahanshahi A. A., Alvarez-Risco, A., Li, J., \& Zhang, S. X. (2020). Anxiety, Distress, and Turnover Intention of Healthcare Workers in Peru by Their Distance to the Epicenter during the COVID-19 Crisis. The American Journal of Tropical Medicine and Hygiene, 103(4), 1614-1620. https://www.ajtmh.org/view/journals/tpmd/103/4/article-p1614.xml https://doi.org/10.4269/ajtmh.20-0800

Yildirim, T. T., Atas, O., Asafov, A., Yildirim, K., \& Balibey, H. (2020). Psychological Status of Healthcare Workers during the Covid-19 Pandemic. Journal of the College of Physicians and Surgeons Pakistan, 30(6), 26-31. https://pubmed.ncbi.nlm.nih.gov/32723444/ https://doi.org/10.29271/jcpsp.2020.supp1.s26 
Research, Society and Development, v. 10, n. 9, e26010918025, 2021

(CC BY 4.0) | ISSN 2525-3409 | DOI: http://dx.doi.org/10.33448/rsd-v10i9.18025

Youssef, N., Mostafa, A., Ezzat, R., Yosef, M., \& Kassas, M. El. (2020). Mental health status of health-care professionals working in quarantine and nonquarantine Egyptian hospitals during the COVID-19 pandemic. Eastern Mediterranean Health Journal, 26(10), 1155-1164. https://applications.emro.who.int/emhj/v26/10/1020-3397-2020-2610-1155-1164-eng.pdf https://doi.org/10.26719/emhj.20.116

Zhang, W. R., Wang, K., Yin, L., Zhao, W. F., Xue, Q., Peng, M., Min, B., Tian, Q., Leng, H., Du, J., Chang, H., Yang, Y., Li, W., Shangguan, F., Yan, T., Dong, H., Han, Y., Wang, Y., Cosci, F., \& Wang, H. (2020). Mental Health and Psychosocial Problems of Medical Health Workers during the COVID-19 Epidemic in China. Psychotherapy and Psychosomatics, 89(4), 242-250. https://www.karger.com/Article/Pdf/507639 https://doi.org/10.1159/000507639

Zhu, W., Wei, Y., Meng, X., \& Li, J. (2020). The mediation effects of coping style on the relationship between social support and anxiety in Chinese medical staff during COVID-19. BMC Health Services Research, 20, 1007. https://bmchealthservres.biomedcentral.com/track/pdf/10.1186/s12913-020-05871-6.pdf https://doi.org/10.1186/s12913-020-05871-6 